\title{
Analytical investigations for heat conduction problems in anisotropic thin-layer media with embedded heat sources
}

\author{
Ming-Hsien Hsieh, Chien-Ching Ma * \\ Department of Mechanical Engineering, National Taiwan University, 1 Roosevelt Road, Sec 4, Taipei 10617, Taiwan, Republic of China
}

Received 23 May 2001; received in revised form 23 March 2002

\begin{abstract}
This article develops the analytical rigorous solution of a fundamental problem of heat conduction in anisotropic media. The steady-state temperature and heat flux fields in a thin-layer medium with anisotropic properties subjected to concentrated embedded heat sources or prescribed temperature on the surface are analyzed. A linear coordinate transformation is used to transform anisotropic thin-layer problems into equivalent isotropic problems without complicating the geometry and boundary conditions of the problem. By using the Fourier transform and the series expansion technique, exact closed-form solutions of the specific problems are presented in series forms. The complete solutions of heat conduction problems for the thin-layer medium consist only of the simplest solutions for an infinite homogeneous medium with concentrated heat sources. The numerical results of the temperature and heat flux distributions are provided in full-field configurations. (c) 2002 Elsevier Science Ltd. All rights reserved.
\end{abstract}

\section{Introduction}

In recent decades, many papers have studied the mechanical behavior of anisotropic materials in applied science and engineering [1]. As a result of interesting usage of anisotropic materials in engineering applications, the analytical solutions for multidimensional heat conduction problems in layered media have attracted considerable attention recently [2]. To date, few investigations of temperature distribution or heat flux fields in anisotropic media have appeared in the open literature. The mathematical difficulties for anisotropic heat conduction problem are caused by the complex form of the governing partial differential equation or by the boundary conditions associated with the problem. A survey of some of the relevant anisotropic heat conduction problems is given by Sharma [3] and Tauchert and Akoz [4]. They solved orthotropic heat conduction problem dealing with infinite or semi-infinite or slab solids by Fourier transformation. Tauchert and Akoz [5] solved the temperature fields of a two-dimensional anisotropic slab using complex conjugate quantities. Mulholland and Gupta [6] investigated a three-dimensional anisotropic body of arbitrary shape by using coordinate transformations to principal axes. Chang [7] solved the heat conduction problem in a three-dimensional configuration by conventional Fourier transformation, and then he indicated that the anisotropic heat conduction governing equation could be transformed to the isotropic one. Poon [8] first surveyed the transformation of heat conduction problems in layered composites from anisotropic to orthotropic. Poon et al. [9] extended coordinate transformation of the anisotropic heat conduction problem to isotropic one. Zhang [10] developed a partition-matching technique to solve a two-dimensional anisotropic strip with prescribed temperature on the boundary.

In earlier papers, analytical solutions of anisotropic heat conduction problems have been limited to simple or special cases [3]. In conventional studies of a multidimensional anisotropic medium subjected to distribute temperature or heat flux in or on the media, the analytical solution was obtained by Fourier transformation. It is not possible to find in most

\footnotetext{
${ }^{*}$ Corresponding author. Tel.: +886-2-2365-9996; fax: +886-2-2363-1755.

E-mail address: cma@w3.me.ntu.edu.tw (C.-C. Ma).
} 


\begin{tabular}{|c|c|c|c|}
\hline \multicolumn{4}{|c|}{ Nomenclature } \\
\hline$c_{p}$ & specific heat $(\mathrm{J} / \mathrm{kg} \mathrm{K})$ & $x_{0}, y_{0}$ & location of the concentrated heat source (m) \\
\hline$f_{x}, f_{y}$ & heat fluxes $\left(\mathrm{W} / \mathrm{m}^{2}\right)$ & $X, Y$ & coordinates in the transform domain (m) \\
\hline & thickness of the slab $(\mathrm{m})$ & \multicolumn{2}{|c|}{ Greek symbols } \\
\hline$H$ & thick of the slab after transformation (m) & $\delta$ & Dirac delta function \\
\hline$K$ & thermal conductivity matrix & & Fourier transform parameter \\
\hline & $\begin{array}{l}\text { thermal conductivity coefficients }(\mathrm{W} / \mathrm{m} \mathrm{K}) \\
\text { coordinate transformation matrix }\end{array}$ & & density $\left(\mathrm{kg} / \mathrm{m}^{3}\right)$ \\
\hline$q_{1}, q_{2}$ & coordinate transformation coefficients & \multirow{2}{*}{\multicolumn{2}{|c|}{$\begin{array}{l}\text { Subscript } \\
m \quad \text { material } 1 \text { or material } 2\end{array}$}} \\
\hline & temperature $(\mathrm{K})$ & & \\
\hline$x, y$ & coordinates (m) & & \\
\hline
\end{tabular}

cases the general solutions with respect to each of the spatial variables to satisfy partial differential equations of anisotropic heat conduction equations and boundary conditions. The work of Yan et al. [11] studied two layered isotropic bodies with homogeneous form of the conduction equation, and the Green function solution was used to incorporate the effects of the internal heat source and nonhomogeneous boundary conditions. They obtained the series solutions for three-dimensional temperature distribution by Fourier transformation, Laplace transformation and eigenvalue methods. Consequently, it is more difficult to get general analytic solutions satisfying all the boundary conditions for multilayered anisotropic heat conduction problem because of the continuity of temperature and heat flux on the interface and the cross-derivatives in the governing equation. Therefore, the cross term, which is the crux in solving the anisotropic heat conduction problem, is very troublesome to treat when one uses conventional solution techniques for solving isotropic heat conduction problems. Due to the mathematical difficulties of the problem, only a few solutions for heat conduction in anisotropic media have appeared in the literature and much more work remains to be done.

In this study, a two-dimensional heat conduction problem for an anisotropic thin-layer medium is investigated. One of the objectives of this study is to develop an effective analytical methodology to construct the full-field solution for this problem. Investigations on anisotropic heat conduction problems are tedious due to the presence of many material constants and the cross derivative term of the governing equation. It is desirable to reduce the dependence on material constants in advance of the analysis of a given boundary value problem. A special linear coordinate transformation is introduced in this study to simplify the governing heat conduction equation without complicating the continuity and boundary conditions of the problem. Based on this transformation, the original anisotropic thin-layer problem is converted to an equivalent isotropic problem with a similar geometrical configuration. Explicit analytical solutions for the temperature and heat flux are expressed in a series form. Numerical results of the full-field distribution for concentrated heat sources in the thin-layer medium or prescribed temperature on the surface are presented in graphic form and discussed in detail.

\section{Basic theory of linear coordinate transformation}

Consider an anisotropic material that is homogeneous and has constant thermo-physical properties. The governing partial differential equation for the heat conduction problem in a two-dimensional anisotropic solid subjected to distributed heat sources of intensity $q\left(x_{i}, t\right)$ is given by

$$
K_{i j} \frac{\partial^{2} T}{\partial x_{i} \partial x_{j}}+\frac{1}{k_{22}} q\left(x_{i}, t\right)=\frac{\rho c_{p}}{k_{22}} \frac{\partial T}{\partial t} \quad i=1,2 \quad j=1,2
$$

in which $\boldsymbol{K}$ is the thermal conductivity matrix

$$
\boldsymbol{K}=\left[\begin{array}{cc}
\frac{k_{11}}{k_{22}} & \frac{k_{12}}{k_{22}} \\
\frac{k_{12}}{k_{22}} & 1
\end{array}\right] \quad K_{i j}=K_{j i}
$$

where $k_{i j}$ are thermal conductivity coefficients. $\rho$ and $c_{p}$ are the density and specific heat, respectively. Based on irreversible thermodynamics, it can be shown that $k_{11} k_{22}>k_{12}^{2}$ in addition to $k_{11}, k_{22}>0$. 
In order to eliminate the cross-derivatives and reduce the governing equation to a canonical form, a special linear coordinate transformation is introduced as

$$
\left\{\begin{array}{l}
X=x+q_{1} y \\
Y=q_{2} y
\end{array} \quad \text { or } \quad \xi=Q \chi\right.
$$

where

$$
\boldsymbol{\xi}=\left[\begin{array}{c}
X \\
Y
\end{array}\right] \quad \chi=\left[\begin{array}{l}
x \\
y
\end{array}\right] \quad \boldsymbol{Q}=\left[\begin{array}{ll}
1 & q_{1} \\
0 & q_{2}
\end{array}\right]
$$

and $\boldsymbol{Q}$ is called the coordinate transformation matrix. The $2 \times 2$ matrix $\boldsymbol{Q}$ should have the following characteristics:

(1) The cross-derivative term of the governing equation can be eliminated.

(2) The governing equation can be converted to an isotropic problem.

(3) In connection with the thin-layer problem, the coordinate transformation matrix $\boldsymbol{Q}$ can keep the two domains remaining perfect contact and bounded interface remaining plane.

(4) In the thin-layer problem, the position along the interface between two adjacent material points maintains the same location and no sliding or mismatching occurs along the interface.

By using the linear coordinate transformation as indicated in Eq. (3), the governing equation becomes

$$
K_{i j} Q_{k i} Q_{s j} \frac{\partial^{2} T}{\partial \xi_{k} \partial \xi_{s}}+\frac{1}{k_{22}} g\left(\xi_{i}, t\right)=\frac{\rho c_{p}}{k_{22}} \frac{\partial T}{\partial t}
$$

It is obvious that if we set

$$
K_{i j} Q_{k i} Q_{s j}=Q_{k i} K_{i j} Q_{j s}^{\mathrm{T}}=c \delta_{k s}
$$

where $c$ is an unknown constant and yet to be determined. Then Eq. (4) can be rewritten to the canonical form as

$$
\frac{\partial^{2} T}{\partial X^{2}}+\frac{\partial^{2} T}{\partial Y^{2}}+\frac{1}{c k_{22}} g(X, Y, t)=\frac{\rho c_{p}}{c k_{22}} \frac{\partial T}{\partial t}
$$

The coordinate transformation matrix $\boldsymbol{Q}$ and the constant $c$ are determined based on Eq. (5) and the results are

$$
\boldsymbol{Q}=\left[\begin{array}{ll}
1 & q_{1} \\
0 & q_{2}
\end{array}\right] \quad q_{1}=-\frac{k_{12}}{k_{22}} \quad q_{2}=\frac{\sqrt{k_{11} k_{22}-k_{12}^{2}}}{k_{22}} \quad c=\frac{k_{11} k_{22}-k_{12}^{2}}{k_{22}^{2}}
$$

\section{Solutions for thin-layer medium subjected to arbitrary thermal loadings}

Consider a steady-state heat conduction problem of an anisotropic thin-layer medium that has a slab with thickness $h$ and is perfectly bounded to a half-plane. The $x$-axis is taken to be the interface. The slab (material 2) occupies $-h<y<0$, while the underlying half-plane occupies $0<y<\infty$. The heat conduction equations and the corresponding heat fluxes in the anisotropic thin-layer medium are

$$
\begin{aligned}
& \frac{k_{11}^{(m)}}{k_{22}^{(m)}} \frac{\partial^{2} T_{m}}{\partial x^{2}}+2 \frac{k_{12}^{(m)}}{k_{22}^{(m)}} \frac{\partial^{2} T_{m}}{\partial x \partial y}+\frac{\partial^{2} T_{m}}{\partial y^{2}}+\frac{1}{k_{22}^{(m)}} g_{m}(x, y)=0 \quad m=1,2 \\
& f_{x}^{(m)}=-k_{11}^{(m)} \frac{\partial T_{m}}{\partial x}-k_{12}^{(m)} \frac{\partial T_{m}}{\partial y} \\
& f_{y}^{(m)}=-k_{12}^{(m)} \frac{\partial T_{m}}{\partial x}-k_{22}^{(m)} \frac{\partial T_{m}}{\partial y}
\end{aligned}
$$

where $g_{m}(x, y)$ are the heat sources inside the medium, $f_{x}^{(m)}$ and $f_{y}^{(m)}$ are the heat fluxes in the $x$ and $y$ directions, respectively.

In numerous cases of practical importance, we consider cases that heat sources act inside the anisotropic thinlayer medium or temperature apply on the boundary. In this article, we will investigate the full-field distributions of 
temperature and heat fluxes due to a concentrated heat source applied in the slab or the half-plane, and a concentrated temperature applied on the top surface of the slab. The temperature and heat flux fields derived for the concentrated heat source problem can be employed in constructing more general solutions concerning distributed sources in a continuous or discontinuous manner in the thin-film medium. The coordinate transformation method developed in Section 2 will be used in the following studies to transform the anisotropic heat conduction problem in a thin-layer medium to an equivalent isotropic problem.

\subsection{Thin-layer medium with a concentrated heat source applied in the slab}

First, let us consider the case that a concentrated heat source is applied in the slab as shown in Fig. 1(a). The concentrated heat source is located at $(x, y)=\left(0,-y_{0}\right)$ and is given by

$$
g_{1}(x, y)=0 \quad g_{2}(x, y)=q_{0} \delta(x) \delta\left(y+y_{0}\right)
$$

The corresponding governing equations of two materials are

$$
\begin{aligned}
& \frac{k_{11}^{(1)}}{k_{22}^{(1)}} \frac{\partial^{2} T_{1}}{\partial x^{2}}+2 \frac{k_{12}^{(1)}}{k_{22}^{(1)}} \frac{\partial^{2} T_{1}}{\partial x \partial y}+\frac{\partial^{2} T_{1}}{\partial y^{2}}=0 \\
& \frac{k_{11}^{(2)}}{k_{22}^{(2)}} \frac{\partial^{2} T_{2}}{\partial x^{2}}+2 \frac{k_{12}^{(2)}}{k_{22}^{(2)}} \frac{\partial^{2} T_{2}}{\partial x \partial y}+\frac{\partial^{2} T_{2}}{\partial y^{2}}+\frac{1}{k_{22}^{(2)}} g_{2}(x, y)=0
\end{aligned}
$$

The boundary condition on the top surface of the slab is

$$
T_{2}(x,-h)=0
$$
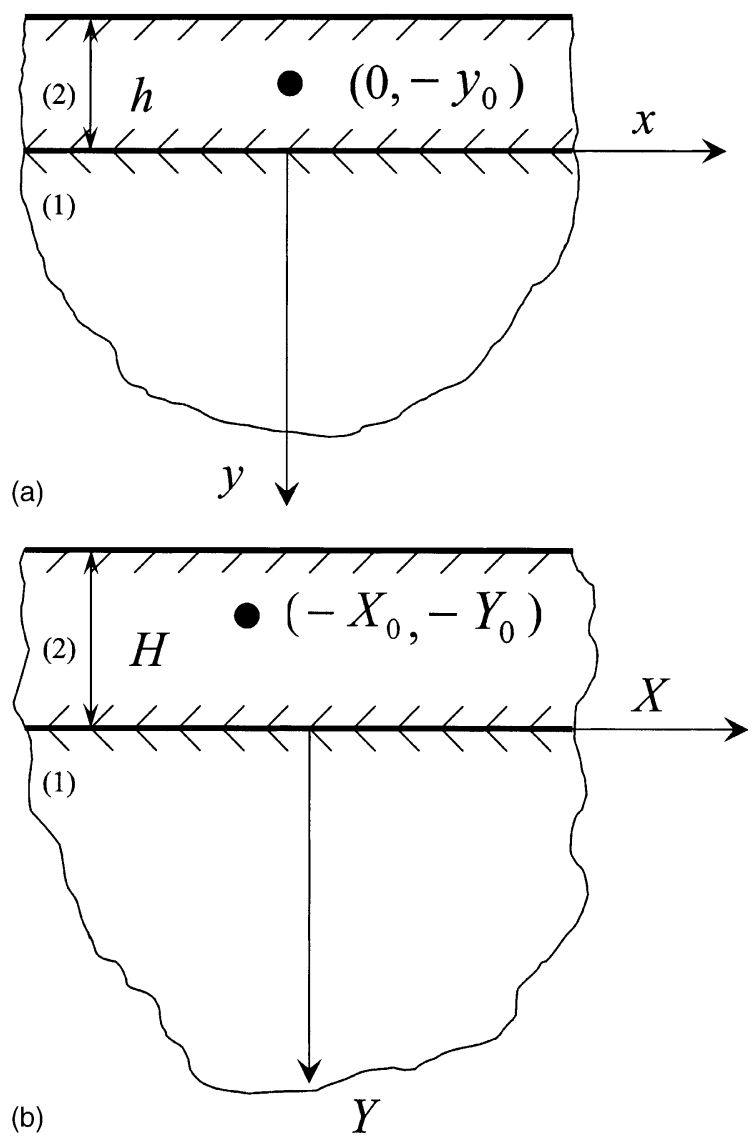

Fig. 1. The geometric configuration of the anisotropic thin-layer medium in the $(x, y)$ coordinate (a) and $(X, Y)$ coordinate (b). 
In addition, perfect thermal contact between the slab and the half-plane is assumed. The continuity of temperature and heat flux along the interface requires

$$
\begin{aligned}
& T_{1}(x, 0)=T_{2}(x, 0) \\
& k_{12}^{(1)} \frac{\partial T_{1}}{\partial x}+k_{22}^{(1)} \frac{\partial T_{1}}{\partial y}=k_{12}^{(2)} \frac{\partial T_{2}}{\partial x}+k_{22}^{(2)} \frac{\partial T_{2}}{\partial y} \quad y=0
\end{aligned}
$$

By introducing the linear coordinate transformation

$$
\boldsymbol{\xi}^{(m)}=\boldsymbol{Q}^{(m)} \boldsymbol{\chi}^{(m)} \quad m=1,2
$$

The boundary value problem as indicated in Eqs. (12)-(16) can be transformed to the $(X, Y)$ coordinate system as

$$
\begin{aligned}
& \frac{\partial^{2} T_{1}}{\partial X^{2}}+\frac{\partial^{2} T_{1}}{\partial Y^{2}}=0 \\
& \frac{\partial^{2} T_{2}}{\partial X^{2}}+\frac{\partial^{2} T_{2}}{\partial Y^{2}}+\frac{q_{0}}{c_{2} k_{22}^{(2)}} \delta\left(X+X_{0}\right) \delta\left(Y+Y_{0}\right)=0 \\
& T_{2}(X,-H)=0 \\
& T_{1}(X, 0)=T_{2}(X, 0) \\
& k_{22}^{(1)} q_{2}^{(1)} \frac{\partial T_{1}}{\partial Y}=k_{22}^{(2)} q_{2}^{(2)} \frac{\partial T_{2}}{\partial Y}
\end{aligned}
$$

where

$$
c_{2}=\frac{k_{11}^{(2)} k_{22}^{(2)}-\left(k_{12}^{(2)}\right)^{2}}{\left(k_{22}^{(2)}\right)^{2}}
$$

and $H=q_{2}^{(2)} h, X_{0}=q_{1}^{(2)} y_{0}, Y_{0}=q_{2}^{(2)} y_{0}$. The solutions of two boundary value problem; i.e. Eqs. (12)-(16) and (18)-(22), are identical to each other from the connection of Eq. (17). The location of the concentrated heat source has been shifted to $(X, Y)=\left(-X_{0},-Y_{0}\right)$. The thickness of the slab is changed from $h$ to $H$ but the constant temperature boundary still remains on a straight line $Y=-H$. Furthermore, the new perfect thermal contact interface between the slab and the half-plane remains a straight line $Y=0$ and the continuity condition of heat flux has been simplified from Eqs. (16)(22). The boundary value problem described by Eqs. (18)-(22) is similar to the thin-layer problem for an isotropic material with a geometric configuration as shown in Fig. 1(b).

The boundary value problem indicated in Eqs. (18)-(22) will be solved by the integral transform technique. The expressions for the field variables will be found by applying a Fourier transform over the spatial coordinate $X$ with parameter $\omega$. The transform pairs for an arbitrary function $f(X, Y)$ is defined as

$$
\hat{f}(\omega, Y)=\int_{-\infty}^{\infty} f(X, Y) \mathrm{e}^{-\mathrm{i} \omega X} \mathrm{~d} X \quad f(X, Y)=\frac{1}{2 \pi} \int_{-\infty}^{\infty} \hat{f}(\omega, Y) \mathrm{e}^{\mathrm{i} \omega X} \mathrm{~d} \omega
$$

where $\mathrm{i}=\sqrt{-1}$.

Apply the Fourier transform to Eqs. (18)-(22) and the results are

$$
\begin{aligned}
& \frac{\partial^{2} \widehat{T}_{1}}{\partial Y^{2}}-\omega^{2} \widehat{T}_{1}=0 \\
& \frac{\partial^{2} \widehat{T}_{2}}{\partial Y^{2}}-\omega^{2} \widehat{T}_{2}=-q_{0} \frac{\delta\left(Y+Y_{0}\right)}{c_{2} k_{22}^{(2)}} \mathrm{e}^{\mathrm{i} \omega X_{0}} \\
& \widehat{T}_{2}(\omega,-H)=0 \\
& \widehat{T}_{1}(\omega, 0)=\widehat{T}_{2}(\omega, 0) \\
& k_{22}^{(1)} q_{2}^{(1)} \frac{\partial \widehat{T}_{1}}{\partial Y}=k_{22}^{(2)} q_{2}^{(2)} \frac{\partial \widehat{T}_{2}}{\partial Y} \quad Y=0
\end{aligned}
$$

The solutions of temperature fields in the Fourier transformed domain can be solved to obtain 


$$
\begin{aligned}
& \widehat{T}_{1}(\omega, Y)=H_{1} \mathrm{e}^{-\omega Y} \\
& \widehat{T}_{2}(\omega, Y)=H_{2} \mathrm{e}^{-\omega Y}+H_{3} \mathrm{e}^{\omega Y}
\end{aligned}
$$

where

$$
\begin{aligned}
& H_{1}=\frac{q_{0} \mathrm{e}^{\mathrm{i} \omega X_{0}}}{2 c_{2} k_{22}^{(2)} \omega} \frac{\mathrm{e}^{-\omega Y_{0}}-\mathrm{e}^{-\omega\left(2 H-Y_{0}\right)}}{\beta_{2}+\beta_{1} \mathrm{e}^{-2 \omega H}} \\
& H_{2}=-\frac{q_{0} \mathrm{e}^{i \omega X_{0}}}{2 c_{2} k_{22}^{(2)} \omega} \frac{\beta_{2} \mathrm{e}^{\omega Y_{0}}+\beta_{1} \mathrm{e}^{-\omega Y_{0}}}{\beta_{2}+\beta_{1} \mathrm{e}^{-2 \omega H}} \mathrm{e}^{-2 \omega H} \\
& H_{3}=\frac{q_{0} \mathrm{e}^{\mathrm{i} \omega X_{0}}}{2 c_{2} k_{22}^{(2)} \omega} \frac{\beta_{2} \mathrm{e}^{\omega Y_{0}}+\beta_{1} \mathrm{e}^{-\omega Y_{0}}}{\beta_{2}+\beta_{1} \mathrm{e}^{-2 \omega H}}
\end{aligned}
$$

in which

$$
\beta_{1}=\frac{1}{2}\left(1-\frac{k_{22}^{(1)} q_{2}^{(1)}}{k_{22}^{(2)} q_{2}^{(2)}}\right) \quad \beta_{2}=\frac{1}{2}\left(1+\frac{k_{22}^{(1)} q_{2}^{(1)}}{k_{22}^{(2)} q_{2}^{(2)}}\right)
$$

with the condition that $\beta_{2}>\beta_{1}$. Because of the term $\beta_{2}+\beta_{1} \mathrm{e}^{-2 \omega H}$ in the denominators of Eqs. (32)-(34), it is impossible to invert the Fourier transform directly. The Taylor series expansion is used which results

$$
\frac{1}{\beta_{2}+\beta_{1} \mathrm{e}^{-2 \omega H}}=\frac{1}{\beta_{2}} \sum_{n=0}^{\infty}(-1)^{n}\left(\frac{\beta_{1}}{\beta_{2}}\right)^{n} \mathrm{e}^{-2 n \omega H}
$$

The temperature filed of the thin-layer medium in the transformed domain can be rewritten as

$$
\begin{aligned}
\widehat{T}_{1}= & \frac{q_{0} \mathrm{e}^{\mathrm{i} \omega X_{0}}}{2 c_{2} k_{22}^{(2)} \omega} \frac{1}{\beta_{2}} \sum_{n=0}^{\infty}(-1)^{n}\left(\frac{\beta_{1}}{\beta_{2}}\right)^{n}\left[\mathrm{e}^{-\omega\left(Y+Y_{0}+2 n H\right)}-\mathrm{e}^{-\omega\left[Y-Y_{0}+2(n+1) H\right]}\right] \\
\widehat{T}_{2}= & \frac{q_{0} \mathrm{e}^{\mathrm{i} \omega X_{0}}}{2 c_{2} k_{22}^{(2)} \omega}\left[\sum_{n=0}^{\infty}(-1)^{n}\left(\frac{\beta_{1}}{\beta_{2}}\right)^{n} \mathrm{e}^{-\omega\left(\left|Y+Y_{0}\right|+2 n H\right)}+\sum_{n=0}^{\infty}(-1)^{n}\left(\frac{\beta_{1}}{\beta_{2}}\right)^{n+1} \mathrm{e}^{-\omega\left(\left|Y-Y_{0}\right|+2 n H\right)}\right. \\
& \left.\quad-\sum_{n=0}^{\infty}(-1)^{n}\left(\frac{\beta_{1}}{\beta_{2}}\right)^{n} \mathrm{e}^{-\omega\left[-\left|Y-Y_{0}\right|+2(n+1) H\right]}-\sum_{n=0}^{\infty}(-1)^{n}\left(\frac{\beta_{1}}{\beta_{2}}\right)^{n+1} \mathrm{e}^{-\omega\left[-\left|Y+Y_{0}\right|+2(n+1) H\right]}\right]
\end{aligned}
$$

Since the solutions in the transformed domain expressed in Eqs. (37) and (38) are mainly exponential functions of $\omega$, the Fourier inverse transformation can be performed term by term. Take the Fourier inverse transformation of Eqs. (37) and (38) and substitute coordinate transformation relation Eq. (17), then we get the exact full-field steady state temperature distributions in the thin-film medium expressed as follows

$$
\begin{aligned}
T_{1}= & -\frac{q_{0}}{4 \pi c_{2} k_{22}^{(2)}} \frac{1}{\beta_{2}} \sum_{n=0}^{\infty}(-1)^{n}\left(\frac{\beta_{1}}{\beta_{2}}\right)^{n} \ln \left\{\left[x+q_{1}^{(1)} y+q_{1}^{(2)} y_{0}\right]^{2}+\left[q_{2}^{(1)} y+q_{2}^{(2)}\left(y_{0}+2 n h\right)\right]^{2}\right\} \\
& +\frac{q_{0}}{4 \pi c_{2} k_{22}^{(2)}} \frac{1}{\beta_{2}} \sum_{n=0}^{\infty}(-1)^{n}\left(\frac{\beta_{1}}{\beta_{2}}\right)^{n} \ln \left\{\left[x+q_{1}^{(1)} y+q_{1}^{(2)} y_{0}\right]^{2}+\left[q_{2}^{(1)} y-q_{2}^{(2)}\left(y_{0}-2(n+1) h\right)\right]^{2}\right\} \\
T_{2}= & -\frac{q_{0}}{4 \pi c_{2} k_{22}^{(2)}} \sum_{n=0}^{\infty}(-1)^{n}\left(\frac{\beta_{1}}{\beta_{2}}\right)^{n} \ln \left\{\left[x+q_{1}^{(2)}\left(y+y_{0}\right)\right]^{2}+\left[q_{2}^{(2)}\left(\left|y+y_{0}\right|+2 n h\right)\right]^{2}\right\}-\frac{q_{0}}{4 \pi c_{2} k_{22}^{(2)}} \sum_{n=0}^{\infty}(-1)^{n}\left(\frac{\beta_{1}}{\beta_{2}}\right)^{n+1} \\
& \times \ln \left\{\left[x+q_{1}^{(2)}\left(y+y_{0}\right)\right]^{2}+\left[q_{2}^{(2)}\left(\left|y-y_{0}\right|+2 n h\right)\right]^{2}\right\}+\frac{q_{0}}{4 \pi c_{2} k_{22}^{(2)}} \sum_{n=0}^{\infty}(-1)^{n}\left(\frac{\beta_{1}}{\beta_{2}}\right)^{n} \ln \left\{\left[x+q_{1}^{(2)}\left(y+y_{0}\right)\right]^{2}\right. \\
& \left.+\left[q_{2}^{(2)}\left(\left|y-y_{0}\right|-2(n+1) h\right)\right]^{2}\right\}+\frac{q_{0}}{4 \pi c_{2} k_{22}^{(2)}} \sum_{n=0}^{\infty}(-1)^{n}\left(\frac{\beta_{1}}{\beta_{2}}\right)^{n+1} \ln \left\{\left[x+q_{1}^{(2)}\left(y+y_{0}\right)\right]^{2}\right. \\
& \left.+\left[q_{2}^{(2)}\left(\left|y+y_{0}\right|-2(n+1) h\right)\right]^{2}\right\}
\end{aligned}
$$


In Eqs. (39) and (40), it is interesting to note that each term in the infinite series represents the solution for a concentrated heat source $q_{0}$ in an infinite homogeneous anisotropic body. Thus the temperature fields of the slab or the half-plane are the summation of the contributions by concentrated heat sources located at different positions in an infinite medium. It is found that the locations of all the heat sources will pass through straight lines $x+q_{1}^{(1)} y+q_{1}^{(2)} y_{0}=0$ and $x+q_{1}^{(2)}\left(y+y_{0}\right)=0$. There is only one term in the infinite series of Eq. (40) representing the applied concentrated heat source $q_{0}$ in the infinite medium at $(x, y)=\left(0,-y_{0}\right)$. All the remaining terms are image concentrated heat sources that are induced to satisfy the boundary and interface conditions. Therefore, the mathematical method used in this study is referred to as the method of images. The advantage of this method is that the solutions of heat conduction problem with complicated geometric configuration can be obtained by superposing the simplest solution in an infinite medium. It is noted that the locations of the image heat sources depend only on the material properties and the geometry of the thin-layer medium.

The corresponding heat flux $f_{y}$ is represented as follows

$$
\begin{aligned}
f_{y}^{(1)}=- & \frac{q_{0}}{2 \pi} \frac{q_{2}^{(1)} k_{22}^{(1)}}{c_{2} k_{22}^{(2)}} \frac{1}{\beta_{2}} \sum_{n=0}^{\infty}(-1)^{n}\left(\frac{\beta_{1}}{\beta_{2}}\right)^{n}\left\{\frac{-\left|q_{2}^{(1)} y+q_{2}^{(2)}\left(y_{0}+2 n h\right)\right|}{\left(x+q_{1}^{(1)} y+q_{1}^{(2)} y_{0}\right)^{2}+\left[q_{2}^{(1)} y+q_{2}^{(2)}\left(y_{0}+2 n h\right)\right]^{2}}\right. \\
& \left.+\frac{\left|q_{2}^{(1)} y-q_{2}^{(2)}\left[y_{0}-2(n+1) h\right]\right|}{\left(x+q_{1}^{(1)} y+q_{1}^{(2)} y_{0}\right)^{2}+\left\{q_{2}^{(1)} y-q_{2}^{(2)}\left[y_{0}-2(n+1) h\right]\right\}^{2}}\right\} \\
f_{y}^{(2)}=- & \frac{q_{0}}{2 \pi} \frac{q_{2}^{(2)}}{c_{2}}\left\{\sum _ { n = 0 } ^ { \infty } ( - 1 ) ^ { n } ( \frac { \beta _ { 1 } } { \beta _ { 2 } } ) ^ { n } \left[\frac{\mp\left|q_{2}^{(2)}\left[\left|y+y_{0}\right|+2 n h\right]\right|}{\left[x+q_{1}^{(2)}\left(y+y_{0}\right)\right]^{2}+\left\{q_{2}^{(2)}\left[\left|y+y_{0}\right|+2 n h\right]\right\}^{2}}\right.\right. \\
& \left.+\frac{\left|q_{2}^{(2)}\left[\left(y-y_{0}\right)+2(n+1) h\right]\right|}{\left[x+q_{1}^{(2)}\left(y+y_{0}\right)\right]^{2}+\left\{q_{2}^{(2)}\left[\left(y-y_{0}\right)+2(n+1) h\right]\right\}^{2}}\right]+\sum_{n=0}^{\infty}(-1)^{n}\left(\frac{\beta_{1}}{\beta_{2}}\right)^{n+1} \\
\times & {\left.\left[\frac{\left|q_{2}^{(2)}\left[\left|y-y_{0}\right|+2 n h\right]\right|}{\left[x+q_{1}^{(2)}\left(y+y_{0}\right)\right]^{2}+\left\{q_{2}^{(2)}\left[\left|y-y_{0}\right|+2 n h\right]\right\}^{2}} \mp \frac{\left.1 y+y_{0} \mid+2(n+1) h\right] \mid}{\left[x+q_{1}^{(2)}\left(y+y_{0}\right)\right]^{2}+\left\{q_{2}^{(2)}\left[-\left|y+y_{0}\right|+2(n+1) h\right]\right\}^{2}}\right]\right\} }
\end{aligned}
$$

where upper symbol in the terms with $\mp$ is for the situation $0 \geqslant y>-y_{0}$, and lower symbols are for $-y_{0}>y>-h$, respectively. For the isotropic thin-layer medium, $k_{11}^{(1)}=k_{22}^{(1)}, k_{11}^{(2)}=k_{22}^{(2)}$ and $k_{12}^{(1)}=k_{12}^{(2)}=0$, hence $q_{1}^{(1)}=q_{1}^{(2)}=0$ and $q_{2}^{(1)}=q_{2}^{(2)}=1$, Eqs. (39)-(42) are reduced to the correspondent isotropic solutions.

If we set the heat conductivity coefficients of the half-plane to the following conditions

$$
k_{11}^{(1)} \rightarrow 0 \quad k_{22}^{(1)} \rightarrow 0 \quad k_{12}^{(1)} \rightarrow 0
$$

then the problem will be reduced to the anisotropic slab with embedded point heat source and insulated on the bottom of the slab. From Eqs. (7) and (35) and results

$$
\beta_{1}=\beta_{2}=\frac{1}{2}
$$

The temperature and heat flux fields in the slab can be obtained from Eqs. (40) and (42) as follows

$$
\begin{aligned}
T_{2}=- & \frac{q_{0}}{4 \pi c_{2} k_{22}^{(2)}}\left[\sum_{n=0}^{\infty}(-1)^{n} \ln \frac{\left\{\left[x+q_{1}^{(2)}\left(y+y_{0}\right)\right]^{2}+\left[q_{2}^{(2)}\left(\left|y+y_{0}\right|+2 n h\right)\right]^{2}\right\}}{\left\{\left[x+q_{1}^{(2)}\left(y+y_{0}\right)\right]^{2}+\left[q_{2}^{(2)}\left(\left|y-y_{0}\right|-2(n+1) h\right)\right]^{2}\right\}}\right. \\
& \left.+\sum_{n=0}^{\infty}(-1)^{n} \ln \frac{\left\{\left[x+q_{1}^{(2)}\left(y+y_{0}\right)\right]^{2}+\left[q_{2}^{(2)}\left(\left|y-y_{0}\right|+2 n h\right)\right]^{2}\right\}}{\left\{\left[x+q_{1}^{(2)}\left(y+y_{0}\right)\right]^{2}+\left[q_{2}^{(2)}\left(\left|y+y_{0}\right|-2(n+1) h\right)\right]^{2}\right\}}\right]
\end{aligned}
$$




$$
\begin{aligned}
f_{y}^{(2)}=- & \frac{q_{0}}{2 \pi} \frac{q_{2}^{(2)}}{c_{2}}\left\{\sum _ { n = 0 } ^ { \infty } ( - 1 ) ^ { n } \left[\frac{\mp\left|q_{2}^{(2)}\left[\left|y+y_{0}\right|+2 n h\right]\right|}{\left[x+q_{1}^{(2)}\left(y+y_{0}\right)\right]^{2}+\left\{q_{2}^{(2)}\left[\left|y+y_{0}\right|+2 n h\right]\right\}^{2}}\right.\right. \\
& \left.+\frac{\left|q_{2}^{(2)}\left[\left(y-y_{0}\right)+2(n+1) h\right]\right|}{\left[x+q_{1}^{(2)}\left(y+y_{0}\right)\right]^{2}+\left\{q_{2}^{(2)}\left[\left(y-y_{0}\right)+2(n+1) h\right]\right\}^{2}}\right]+\sum_{n=0}^{\infty}(-1)^{n}\left[\frac{\left|q_{2}^{(2)}\left[\left|y-y_{0}\right|+2 n h\right]\right|}{\left[x+q_{1}^{(2)}\left(y+y_{0}\right)\right]^{2}+\left\{q_{2}^{(2)}\left[\left|y-y_{0}\right|+2 n h\right]\right\}^{2}}\right. \\
& \left.\left.\mp \frac{\left|q_{2}^{(2)}\left[-\left|y+y_{0}\right|+2(n+1) h\right]\right|}{\left[x+q_{1}^{(2)}\left(y+y_{0}\right)\right]^{2}+\left\{q_{2}^{(2)}\left[-\left|y+y_{0}\right|+2(n+1) h\right]\right\}^{2}}\right]\right\}
\end{aligned}
$$

It is interesting to note that the infinite series solutions presented in Eqs. (45) and (46) can be expressed explicitly with simple functional forms as follows

$$
\begin{aligned}
T_{2}=- & \frac{q_{0}}{8 \pi c_{2} k_{22}^{(2)}}\left\{\ln \left[-\cosh \frac{\pi}{2 q_{2}^{(2)} h}\left(x+q_{1}^{(2)}\left(y-h-y_{0}\right)\right)+\cos \frac{\pi}{2 h}\left(y-h-y_{0}\right)\right]\right. \\
& -\ln \left[\cosh \frac{\pi}{2 q_{2}^{(2)} h}\left(x+q_{1}^{(2)}\left(y-h-y_{0}\right)\right)+\cos \frac{\pi}{2 h}\left(y-h-y_{0}\right)\right]+\ln \left[-\cosh \frac{\pi}{2 q_{2}^{(2)} h}\left(x+q_{1}^{(2)}\left(y-h-y_{0}\right)\right)\right. \\
& \left.\left.+\cos \frac{\pi}{2 h}\left(y-h+y_{0}\right)\right]-\ln \left[\cosh \frac{\pi}{2 q_{2}^{(2)} h}\left(x+q_{1}^{(2)}\left(y-h-y_{0}\right)\right)+\cos \frac{\pi}{2 h}\left(y-h+y_{0}\right)\right]\right\} \\
f_{y}^{(2)}= & \frac{q_{0} q_{2}^{(2)}}{4 c_{2}} \cosh \left(\frac{\pi}{2 h} x\right)\left\{\frac{\sin (\pi / 2 h)\left(y-h-y_{0}\right)}{\cosh \left(\pi / q_{2}^{(2)} h\right)\left[x+q_{1}^{(2)}\left(y-h-y_{0}\right)\right]-\cos (\pi / h)\left(y-h-y_{0}\right)}\right. \\
& \left.+\frac{\sin (\pi / 2 h)\left(y-h+y_{0}\right)}{\cosh \left(\pi / q_{2}^{(2)} h\right)\left[x+q_{1}^{(2)}\left(y-h-y_{0}\right)\right]-\cos (\pi / h)\left(y-h+y_{0}\right)}\right\}
\end{aligned}
$$

\subsection{Thin-layer medium with a concentrated heat source applied in the half-plane}

The problem for a concentrated heat source $q_{0}$ applied in the half-plane is considered. The location of the concentrated heat source in the half-plane is $(x, y)=\left(0, y_{0}\right)$. Hence we have

$$
g_{1}(x, y)=q_{0} \delta(x) \delta\left(y-y_{0}\right) \quad g_{2}(x, y)=0
$$

and the partial differential equations of the problem are

$$
\begin{aligned}
& \frac{k_{11}^{(1)}}{k_{22}^{(1)}} \frac{\partial^{2} T_{1}}{\partial x^{2}}+2 \frac{k_{12}^{(1)}}{k_{22}^{(1)}} \frac{\partial^{2} T_{1}}{\partial x \partial y}+\frac{\partial^{2} T_{1}}{\partial y^{2}}+\frac{1}{k_{22}^{(1)}} g_{1}(x, y)=0 \\
& \frac{k_{11}^{(2)}}{k_{22}^{(2)}} \frac{\partial^{2} T_{2}}{\partial x^{2}}+2 \frac{k_{12}^{(2)}}{k_{22}^{(2)}} \frac{\partial^{2} T_{2}}{\partial x \partial y}+\frac{\partial^{2} T_{2}}{\partial y^{2}}=0
\end{aligned}
$$

If the temperature on the surface of the slab vanishes, the boundary condition is

$$
T_{2}(x,-h)=0
$$

The perfect thermal contact condition between slab and half-plane is also assumed. Thus at the interface of the thinlayer, the continuity conditions in Eqs. (15) and (16) are used. Follow the similar procedure that is used in the previous case, the linear coordinate transformation (Eq. (17)) is introduced and the governing equations in the $(X, Y)$ coordinate are

$$
\frac{\partial^{2} T_{1}}{\partial X^{2}}+\frac{\partial^{2} T_{1}}{\partial Y^{2}}+\frac{q_{0}}{c_{1} k_{22}^{(1)}} \delta\left(X-X_{0}\right) \delta\left(Y-Y_{0}\right)=0
$$




$$
\frac{\partial^{2} T_{2}}{\partial X^{2}}+\frac{\partial^{2} T_{2}}{\partial Y^{2}}=0
$$

where

$$
c_{1}=\frac{k_{11}^{(1)} k_{22}^{(1)}-\left(k_{12}^{(1)}\right)^{2}}{\left(k_{22}^{(1)}\right)^{2}}
$$

and $X_{0}=q_{1}^{(1)} y_{0}, Y_{0}=q_{2}^{(1)} y_{0}$. The boundary and continuity conditions are the same as those given in Eqs. (20)-(22). Use the method as described in the previous case, and the complete temperature solutions in the transformed domain are

$$
\begin{aligned}
\widehat{T}_{1}= & \frac{q_{0} \mathrm{e}^{-\mathrm{i} \omega X_{0}}}{2 c_{1} k_{22}^{(1)} \omega} \mathrm{e}^{-\omega\left|Y-Y_{0}\right|}+\frac{q_{0} \mathrm{e}^{-\mathrm{i} \omega X_{0}}}{2 c_{1} k_{22}^{(1)} \omega} \sum_{n=0}^{\infty}\left(\frac{\eta-1}{\eta+1}\right)^{n+1} \mathrm{e}^{-\omega\left(Y+Y_{0}+2 n H\right)}-\frac{q_{0} \mathrm{e}^{-\mathrm{i} \omega X_{0}}}{2 c_{1} k_{22}^{(1)} \omega} \sum_{n=0}^{\infty}\left(\frac{\eta-1}{\eta+1}\right)^{n} \mathrm{e}^{-\omega\left[Y+Y_{0}+2(n+1) H\right]} \\
\widehat{T}_{2}= & \frac{q_{0} \mathrm{e}^{-\mathrm{i} \omega X_{0}}}{4 c_{1} k_{22}^{(1)} \omega}(\eta+1)\left[\mathrm{e}^{-\omega\left|Y-Y_{0}\right|}+\sum_{n=0}^{\infty}\left(\frac{\eta-1}{\eta+1}\right)^{n+1} \mathrm{e}^{-\omega\left|Y+Y_{0}+2 n H\right|}-\sum_{n=0}^{\infty}\left(\frac{\eta-1}{\eta+1}\right)^{n} \mathrm{e}^{-\omega\left|Y+Y_{0}+2(n+1) H\right|}\right] \\
& -\frac{q_{0} \mathrm{e}^{-\mathrm{i} \omega X_{0}}}{4 c_{1} k_{22}^{(1)} \omega}(\eta-1)\left[\mathrm{e}^{-\omega\left|Y+Y_{0}\right|}+\sum_{n=0}^{\infty}\left(\frac{\eta-1}{\eta+1}\right)^{n+1} \mathrm{e}^{-\omega\left|Y_{0}-Y+2 n H\right|}-\sum_{n=0}^{\infty}\left(\frac{\eta-1}{\eta+1}\right)^{n} \mathrm{e}^{-\omega\left|Y_{0}-Y+2(n+1) H\right|}\right]
\end{aligned}
$$

where

$$
H=q_{2}^{(2)} h \quad \eta=\frac{k_{22}^{(1)} q_{2}^{(1)}}{k_{22}^{(2)} q_{2}^{(2)}}
$$

The temperature fields in the $(x, y)$ coordinate can be obtained by the Fourier inversed transform term by term and the coordinate transformation relation in Eq. (17). The complete solutions of temperature distribution for the anisotropic thin-layer medium are presented as follows

$$
\begin{aligned}
T_{1}= & -\frac{q_{0}}{4 \pi c_{1} k_{22}^{(1)}}\left[\ln \left\{\left[x+q_{1}^{(1)}\left(y-y_{0}\right)\right]^{2}+\left[q_{2}^{(1)}\left(y-y_{0}\right)\right]^{2}\right\}+\sum_{n=0}^{\infty}\left(\frac{\eta-1}{\eta+1}\right)^{n+1} \ln \left\{\left[x+q_{1}^{(1)}\left(y-y_{0}\right)\right]^{2}+\left[q_{2}^{(1)}\left(y+y_{0}\right)\right.\right.\right. \\
& \left.\left.\left.+2 n q_{2}^{(2)} h\right]^{2}\right\}-\sum_{n=0}^{\infty}\left(\frac{\eta-1}{\eta+1}\right)^{n} \ln \left\{\left[x+q_{1}^{(1)}\left(y-y_{0}\right)\right]^{2}+\left[q_{2}^{(1)}\left(y+y_{0}\right)+2(n+1) q_{2}^{(2)} h\right]^{2}\right\}\right] \\
T_{2}= & -\frac{q_{0}}{8 \pi c_{1} k_{22}^{(1)}}\left\{( \eta + 1 ) \left[\ln \left\{\left(x+q_{1}^{(2)} y-q_{1}^{(1)} y_{0}\right)^{2}+\left(q_{2}^{(2)} y-q_{2}^{(1)} y_{0}\right)^{2}\right\}+\sum_{n=0}^{\infty}\left(\frac{\eta-1}{\eta+1}\right)^{n+1} \ln \left\{\left(x+q_{1}^{(2)} y-q_{1}^{(1)} y_{0}\right)^{2}\right.\right.\right. \\
& \left.\left.\left.+\left[q_{2}^{(2)} y+q_{2}^{(1)} y_{0}+2 n q_{2}^{(2)} h\right]^{2}\right\}-\sum_{n=0}^{\infty}\left(\frac{\eta-1}{\eta+1}\right)^{n} \ln \left\{\left(x+q_{1}^{(2)} y-q_{1}^{(1)} y_{0}\right)^{2}+\left[q_{2}^{(2)} y+q_{2}^{(1)} y_{0}+2(n+1) q_{2}^{(2)} h\right]^{2}\right\}\right]\right\} \\
& +\frac{q_{0}}{8 \pi c_{1} k_{22}^{(1)}}\left\{( \eta - 1 ) \left[\ln \left\{\left(x+q_{1}^{(2)} y-q_{1}^{(1)} y_{0}\right)^{2}+\left(q_{2}^{(2)} y+q_{2}^{(1)} y_{0}\right)^{2}\right\}+\sum_{n=0}^{\infty}\left(\frac{\eta-1}{\eta+1}\right)^{n+1} \ln \left\{\left(x+q_{1}^{(2)} y-q_{1}^{(1)} y_{0}\right)^{2}\right.\right.\right. \\
& \left.\left.\left.+\left[q_{2}^{(1)} y_{0}-q_{2}^{(2)} y+2 n q_{2}^{(2)} h\right]^{2}\right\}-\sum_{n=0}^{\infty}\left(\frac{\eta-1}{\eta+1}\right)^{n} \ln \left\{\left(x+q_{1}^{(2)} y-q_{1}^{(1)} y_{0}\right)^{2}+\left[q_{2}^{(1)} y_{0}-q_{2}^{(2)} y+2(n+1) q_{2}^{(2)} h\right]^{2}\right\}\right]\right\}
\end{aligned}
$$

Note that the first term in Eq. (59) without summation represents the concentrated heat source $q_{0}$ applied in the anisotropic half-plane at $(x, y)=\left(0, y_{0}\right)$, and the summation terms provide the image concentrated heat sources that are induced to satisfy the boundary and interface conditions. Again each term in Eqs. (59) and (60) represents the solution for a concentrated heat source applied in an infinite anisotropic medium.

The corresponding solutions for the heat flux $f_{y}$ can be obtained as 


$$
\begin{aligned}
& f_{y}^{(1)}=-\frac{q_{0}}{2 \pi} \frac{q_{2}^{(1)}}{c_{1}}\left\{ \pm \frac{\left|q_{2}^{(1)}\left(y-y_{0}\right)\right|}{\left[x+q_{1}^{(1)}\left(y-y_{0}\right)\right]^{2}+\left[q_{2}^{(1)}\left(y-y_{0}\right)\right]^{2}}\right. \\
& -\sum_{n=0}^{\infty}\left(\frac{\eta-1}{\eta+1}\right)^{n+1} \frac{\left|q_{2}^{(1)}\left(y+y_{0}\right)+2 n q_{2}^{(2)} h\right|}{\left[x+q_{1}^{(1)}\left(y-y_{0}\right)\right]^{2}+\left[q_{2}^{(1)}\left(y+y_{0}\right)+2 n q_{2}^{(2)} h\right]^{2}} \\
& \left.+\sum_{n=0}^{\infty}\left(\frac{\eta-1}{\eta+1}\right)^{n} \frac{\left|q_{2}^{(1)}\left(y+y_{0}\right)+2(n+1) q_{2}^{(2)} h\right|}{\left[x+q_{1}^{(1)}\left(y-y_{0}\right)\right]^{2}+\left\{q_{2}^{(1)}\left(y+y_{0}\right)+2(n+1) q_{2}^{(2)} h\right\}^{2}}\right\} \\
& f_{y}^{(2)}=-\frac{q_{0}}{4 \pi} \frac{q_{2}^{(2)} k_{22}^{(2)}}{c_{1} k_{22}^{(1)}}\left\{( \eta + 1 ) \left[\frac{\left|q_{2}^{(2)} y-q_{2}^{(1)} y_{0}\right|}{\left[x+q_{1}^{(2)} y-q_{1}^{(1)} y_{0}\right]^{2}+\left[q_{2}^{(2)} y-q_{2}^{(1)} y_{0}\right]^{2}}\right.\right. \\
& -\sum_{n=0}^{\infty}\left(\frac{\eta-1}{\eta+1}\right)^{n+1} \frac{\left|q_{2}^{(2)} y+q_{2}^{(1)} y_{0}+2 n q_{2}^{(2)} h\right|}{\left[x+q_{1}^{(2)} y-q_{1}^{(1)} y_{0}\right]^{2}+\left[q_{2}^{(2)} y+q_{2}^{(1)} y_{0}+2 n q_{2}^{(2)} h\right]^{2}} \\
& \left.+\sum_{n=0}^{\infty}\left(\frac{\eta-1}{\eta+1}\right)^{n} \frac{\left|q_{2}^{(2)} y+q_{2}^{(1)} y_{0}+2(n+1) q_{2}^{(2)} h\right|}{\left[x+q_{1}^{(2)} y-q_{1}^{(1)} y_{0}\right]^{2}+\left[q_{2}^{(2)} y+q_{2}^{(1)} y_{0}+2(n+1) q_{2}^{(2)} h\right]^{2}}\right] \\
& +(\eta-1)\left[\frac{\left|q_{2}^{(2)} y+q_{2}^{(1)} y_{0}\right|}{\left[x+q_{1}^{(2)} y-q_{1}^{(1)} y_{0}\right]^{2}+\left[q_{2}^{(2)} y+q_{2}^{(1)} y_{0}\right]^{2}}\right. \\
& -\sum_{n=0}^{\infty}\left(\frac{\eta-1}{\eta+1}\right)^{n+1} \frac{\left|q_{2}^{(1)} y_{0}-q_{2}^{(2)} y+2 n q_{2}^{(2)} h\right|}{\left[x+q_{1}^{(2)} y-q_{1}^{(1)} y_{0}\right]^{2}+\left[q_{2}^{(1)} y_{0}-q_{2}^{(2)} y+2 n q_{2}^{(2)} h\right]^{2}} \\
& \left.\left.+\sum_{n=0}^{\infty}\left(\frac{\eta-1}{\eta+1}\right)^{n} \frac{\left|q_{2}^{(1)} y_{0}-q_{2}^{(2)} y+2(n+1) q_{2}^{(2)} h\right|}{\left[x+q_{1}^{(2)} y-q_{1}^{(1)} y_{0}\right]^{2}+\left[q_{2}^{(1)} y_{0}-q_{2}^{(2)} y+2(n+1) q_{2}^{(2)} h\right]^{2}}\right]\right\}
\end{aligned}
$$

where the upper symbol \pm in the first term of Eq. (61) is for $y_{0} \geqslant y>0$, and the lower symbol is for $\infty>y>y_{0}$.

\subsection{Thin-layer medium with a prescribed temperature on the surface of the slab}

Consider the problem of a prescribed temperature on the top surface of the slab. The heat conduction equations of the thin-layer medium are given by

$$
k_{11}^{(m)} \frac{\partial^{2} T_{m}}{\partial x^{2}}+2 k_{12}^{(m)} \frac{\partial^{2} T_{m}}{\partial x \partial y}+k_{22}^{(m)} \frac{\partial^{2} T_{m}}{\partial y^{2}}=0 \quad m=1,2
$$

If the temperature distribution along the surface of the slab is a delta function, then

$$
T_{2}(x,-h)=T_{0} \delta\left(x-x_{0}\right)
$$

The perfect thermal contact condition between the slab and the half-plane is also assumed. There are no heat source inside the slab and the half-plane. Consequently, the continuity conditions indicated in Eqs. (15) and (16) are used. By using the coordinate transformation, the associated problem in the $(X, Y)$ coordinate is given as follows

$$
\frac{\partial^{2} T_{m}}{\partial X^{2}}+\frac{\partial^{2} T_{m}}{\partial Y^{2}}=0 \quad m=1,2
$$




$$
\begin{aligned}
& T_{2}(X,-H)=T_{0} \delta\left(X-X_{0}\right) \\
& T_{1}(X, 0)=T_{2}(X, 0) \\
& k_{22}^{(1)} q_{2}^{(1)} \frac{\partial T_{1}}{\partial Y}=k_{22}^{(2)} q_{2}^{(2)} \frac{\partial T_{2}}{\partial Y} \quad Y=0
\end{aligned}
$$

where $H=q_{2}^{(2)} h$ and $X_{0}=x_{0}-q_{1}^{(2)} h$. Apply the Fourier transformation on Eqs. (65)-(68), then we have

$$
\begin{aligned}
& \frac{\partial^{2} \widehat{T}_{m}}{\partial Y^{2}}-\omega^{2} \widehat{T}_{m}=0 \quad m=1,2 \\
& \widehat{T}_{2}(\omega,-H)=T_{0} \mathrm{e}^{\mathrm{i} \omega X_{0}} \\
& \widehat{T}_{1}(\omega, 0)=\widehat{T}_{2}(\omega, 0) \\
& k_{22}^{(1)} q_{2}^{(1)} \frac{\partial \widehat{T}_{1}}{\partial Y}=k_{22}^{(2)} q_{2}^{(2)} \frac{\partial \widehat{T}_{2}}{\partial Y} \quad Y=0
\end{aligned}
$$

The temperature fields in the Fourier transform domain are

$$
\begin{aligned}
& \widehat{T}_{1}=2 T_{0} \mathrm{e}^{\mathrm{i} \omega X_{0}} \frac{1}{\eta+1} \sum_{n=0}^{\infty}\left(\frac{\eta-1}{\eta+1}\right)^{n} \mathrm{e}^{-\omega[Y+(2 n+1) H]} \\
& \widehat{T}_{2}=T_{0} \mathrm{e}^{-\mathrm{i} \omega X_{0}}\left\{\sum_{n=0}^{\infty}\left(\frac{\eta-1}{\eta+1}\right)^{n} \mathrm{e}^{-\omega|Y+(2 n+1) H|}-\sum_{n=0}^{\infty}(-1)^{n}\left(\frac{\eta-1}{\eta+1}\right)^{n+1} \mathrm{e}^{-\omega|Y-(2 n+1) H|}\right\}
\end{aligned}
$$

where

$$
\eta=\frac{k_{22}^{(1)} q_{2}^{(1)}}{k_{22}^{(2)} q_{2}^{(2)}}
$$

The full-field temperature and heat flux distributions in the $(x, y)$ coordinate are represented as follows

$$
\begin{aligned}
T_{1}= & \frac{2 T_{0}}{\pi} \frac{1}{\eta+1} \sum_{n=0}^{\infty}\left(\frac{\eta-1}{\eta+1}\right)^{n} \frac{\left|q_{2}^{(1)} y+(2 n+1) q_{2}^{(2)} h\right|}{\left[\left(x-x_{0}\right)+\left(q_{1}^{(1)} y+q_{1}^{(2)} h\right)\right]^{2}+\left[q_{2}^{(1)} y+(2 n+1) q_{2}^{(2)} h\right]^{2}} \\
T_{2}= & \frac{T_{0}}{\pi}\left\{\sum_{n=0}^{\infty}\left(\frac{\eta-1}{\eta+1}\right)^{n} \frac{\left|q_{2}^{(2)}[y+(2 n+1) h]\right|}{\left[\left(x-x_{0}\right)+q_{1}^{(2)}(y+h)\right]^{2}+\left\{q_{2}^{(2)}[y+(2 n+1) h]\right\}^{2}}\right. \\
& \left.-\sum_{n=0}^{\infty}\left(\frac{\eta-1}{\eta+1}\right)^{n+1} \frac{\left|q_{2}^{(2)}[y-(2 n+1) h]\right|}{\left[\left(x-x_{0}\right)+q_{1}^{(2)}(y+h)\right]^{2}+\left\{q_{2}^{(2)}[y-(2 n+1) h]^{2}\right\}}\right\} \\
f_{y}^{(1)}= & \frac{2 T_{0}}{\pi} \frac{k_{22}^{(1)} q_{2}^{(1)} \sum_{n=0}^{\infty}\left(\frac{\eta-1}{\eta+1}\right)^{n}\left\{\frac{\left\{\left[\left(x-x_{0}\right)+\left(q_{1}^{(1)} y+q_{1}^{(2)} h\right)\right]^{2}+\left[q_{2}^{(1)} y+(2 n+1) q_{2}^{(2)} h\right]^{2}\right.}{\{+1}\right\}^{2}}{\left[\left(x-x_{0}\right)+\left(q_{1}^{(1)} y+q_{1}^{(2)} h\right)\right]^{2}} \\
& \left.-\frac{\left.\left[\left(x-x_{0}\right)+\left(q_{1}^{(1)} y+q_{1}^{(2)} h\right)\right]^{2}+\left[q_{2}^{(1)} y+(2 n+1) q_{2}^{(2)} h\right]^{2}\right\}^{2}}{\left\{{ }^{2}\right.}\right\}
\end{aligned}
$$




$$
\begin{aligned}
f_{y}^{(2)}=\frac{T_{0}}{\pi} k_{22}^{(2)} q_{2}^{(2)} & \left\{\sum _ { n = 0 } ^ { \infty } ( \frac { \eta - 1 } { \eta + 1 } ) ^ { n } \left(\frac{\left[q_{2}^{(2)}[y+(2 n+1) h]\right]^{2}}{\left\{\left[\left(x-x_{0}\right)+q_{1}^{(2)}(y+h)\right]^{2}+\left[q_{2}^{(2)}[y+(2 n+1) h]\right]^{2}\right\}^{2}}\right.\right. \\
& \left.-\frac{\left[\left(x-x_{0}\right)+q_{1}^{(2)}(y+h)\right]^{2}}{\left\{\left[\left(x-x_{0}\right)+q_{1}^{(2)}(y+h)\right]^{2}+\left[q_{2}^{(2)}[y+(2 n+1) h]\right]^{2}\right\}^{2}}\right)^{n}\left[\frac{\left[q_{2}^{(2)}[y-(2 n+1) h]\right]^{2}}{\left\{\left[\left(x-x_{0}\right)+q_{1}^{(2)}(y+h)\right]^{2}+\left[q_{2}^{(2)}[y-(2 n+1) h]\right]^{2}\right\}^{2}}\right. \\
+ & \sum_{n=0}^{\infty}\left(\frac{\eta-1}{\eta+1}\right)^{n+1}\left(\left[x-x_{0}\right)+q_{1}^{(2)}(y+h)\right]^{2} \\
& \left.-\frac{\left.\left[\left(x-x_{0}\right)+q_{1}^{(2)}(y+h)\right]^{2}+\left[q_{2}^{(2)}[y-(2 n+1) h]\right]^{2}\right\}}{\{}\right\}
\end{aligned}
$$

\section{Numerical examples and discussions}

By using the analytical solutions developed in the previous sections in conjunction with a personal computer, accurate numerical calculations of temperature and heat flux in the $y$-direction are obtained via a computer program in a

Table 1

Coefficient of thermal conductivity (W/m K)

\begin{tabular}{llll}
\hline & Material & \\
\cline { 2 - 3 } & 1 & 2 & 76.5 \\
\hline$k_{11}$ & 83.6 & 20.6 & \\
$k_{12}$ & 18.1 & 52.7 & \\
$k_{22}$ & 20.8 & & \\
\hline
\end{tabular}

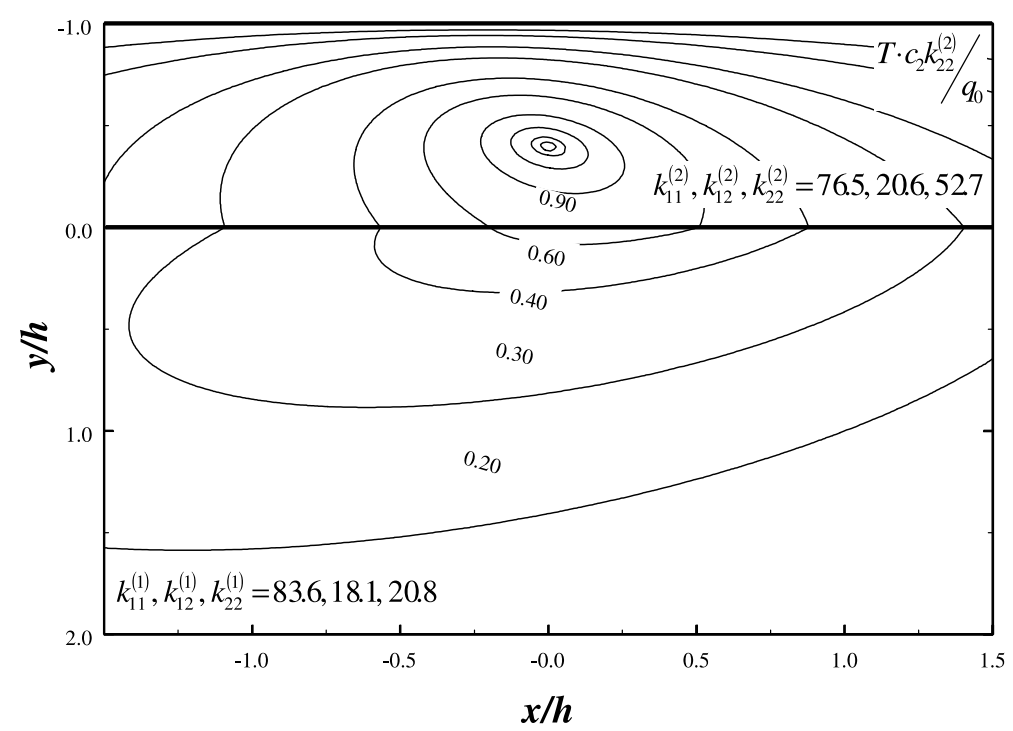

Fig. 2. Temperature distribution of the thin-layer medium with a concentrated heat source applied in the slab. 


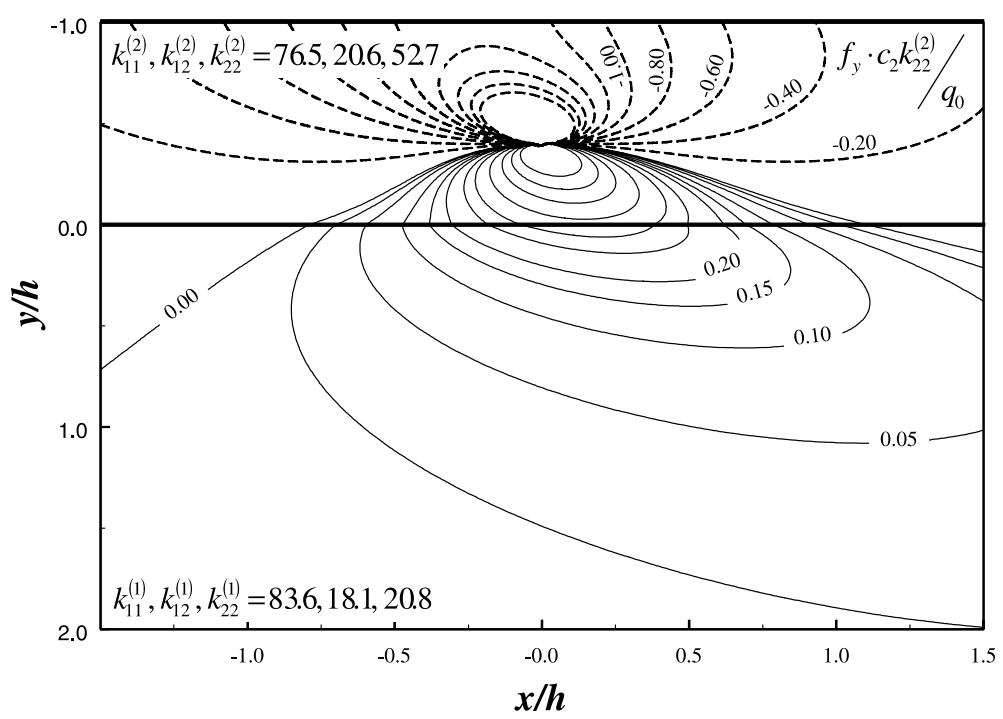

Fig. 3. Heat flux in the $y$-direction of the thin-layer medium with a concentrated heat source applied in the slab.

few minutes. For the purpose of illustrating the thermal anisotropy, an orthorhombic crystal is considered and the pertinent material properties are listed in Table 1 [12]. For the purpose of accurate evaluation of temperature and heat flux fields far away from the heat source, the terms in the series solutions must take a large number. Usually hundred of terms in the series solutions are used for the numerical calculation.

Figs. 2 and 3 show the full-field distributions of temperature and heat flux in the $y$-direction for a concentrated heat source applied within the layer at $(0,-0.4 h)$, respectively. The temperature on the surface of the layer $y=-h$ is vanished. The temperature and the flow of heat in the anisotropic material occur primarily in the $x$-direction owning to the larger thermal conductivity in the direction $\left(k_{11}>k_{22}\right)$. In anisotropic media, the symmetry for the temperature and heat flux fields that is found in the isotropic material is distorted due to the material anisotropy. It is shown in the

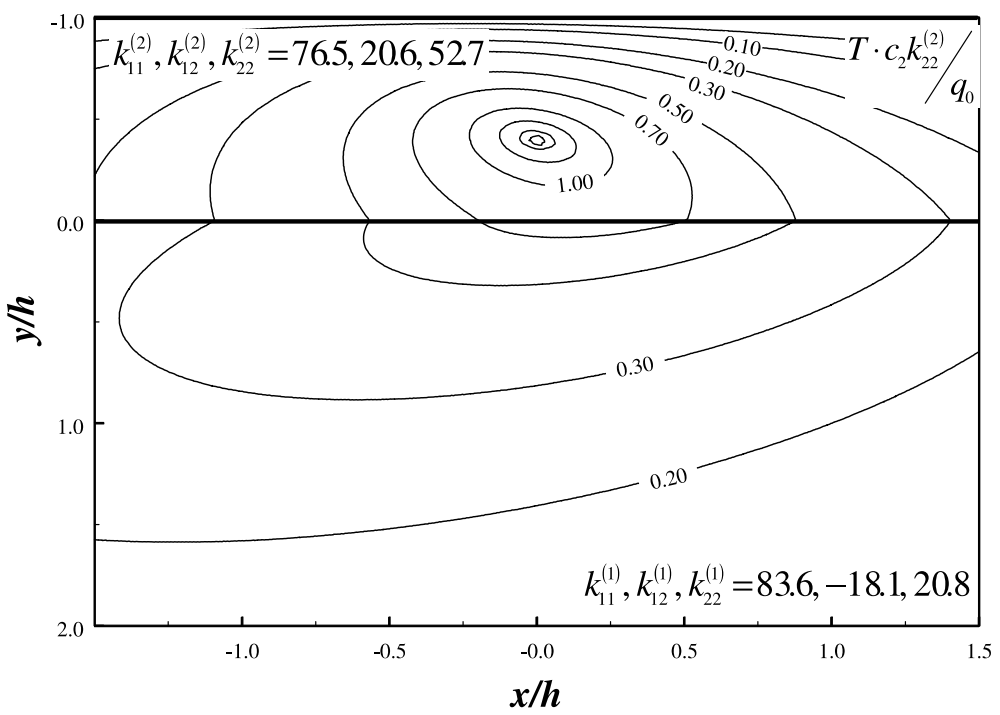

Fig. 4. Temperature distribution of the thin-layer medium with a concentrated heat source applied in the slab. 


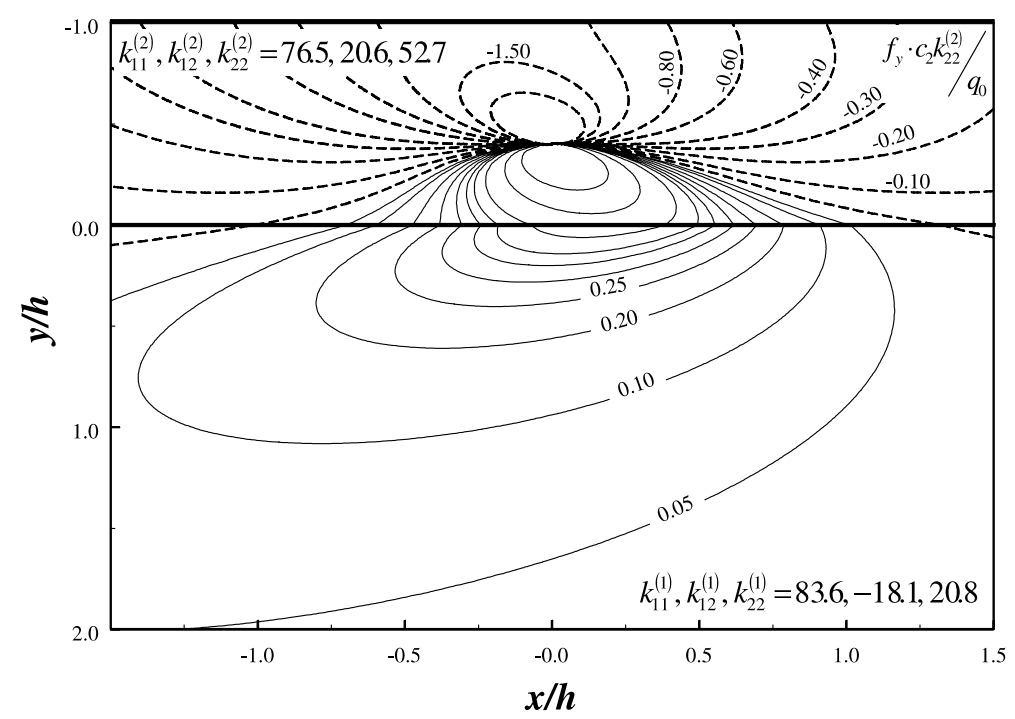

Fig. 5. Heat flux in the $y$-direction of the thin-layer medium with a concentrated heat source applied in the slab.

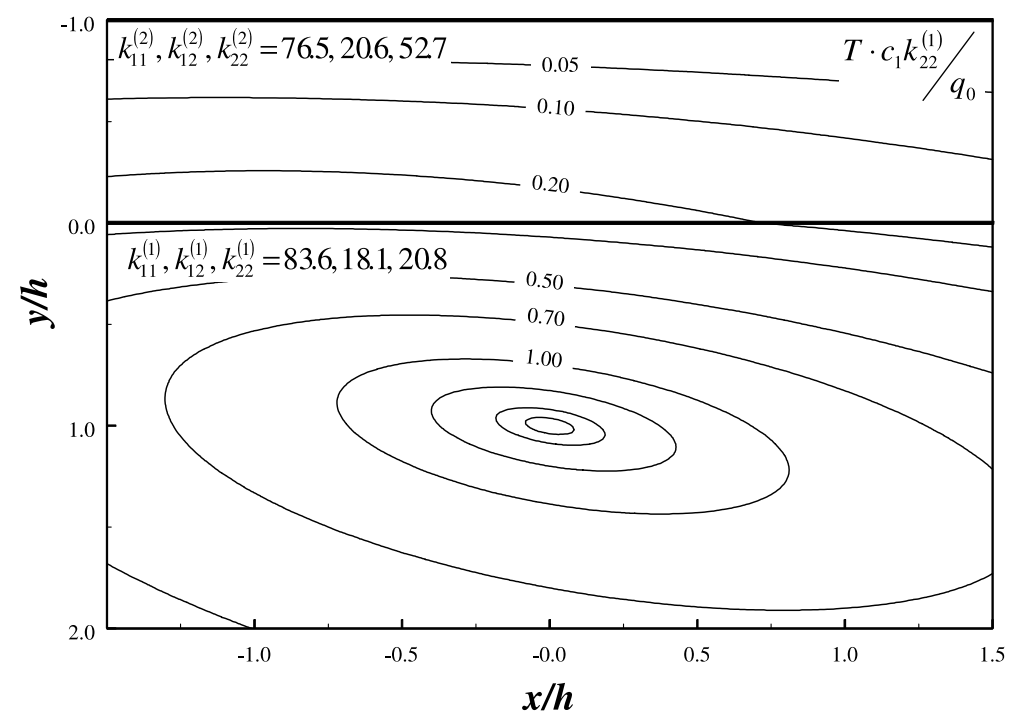

Fig. 6. Temperature distribution of the thin-layer medium with a concentrated heat source applied in the half-plane.

figures that the temperature and heat flux in the $y$-direction are continuous at the interface. This also indicates that the convergence and accuracy for the numerical calculation are satisfied. Figs. 4 and 5 depict the influence of the thermal conductivity coefficient $k_{12}$ on the temperature and heat flux fields. It is evident that the negative value of $k_{12}$ has a marked influence on the temperature and heat flux distributions.

Figs. 6 and 7 are the plots of the temperature distribution and heat flux field in the $y$-direction with a concentrated heat source applied in the half-plane, respectively. The effects of material anisotropy are clearly illustrated in the figures. As noted preciously, the temperature and heat tend to flow away from the hot region in the direction of the greatest thermal conductivity. Figs. 8 and 9 are the full-field distributions of temperature and heat flux in the $y$-direction with a concentrated temperature applied on the top surface of the slab for a thin-layer medium, respectively. 


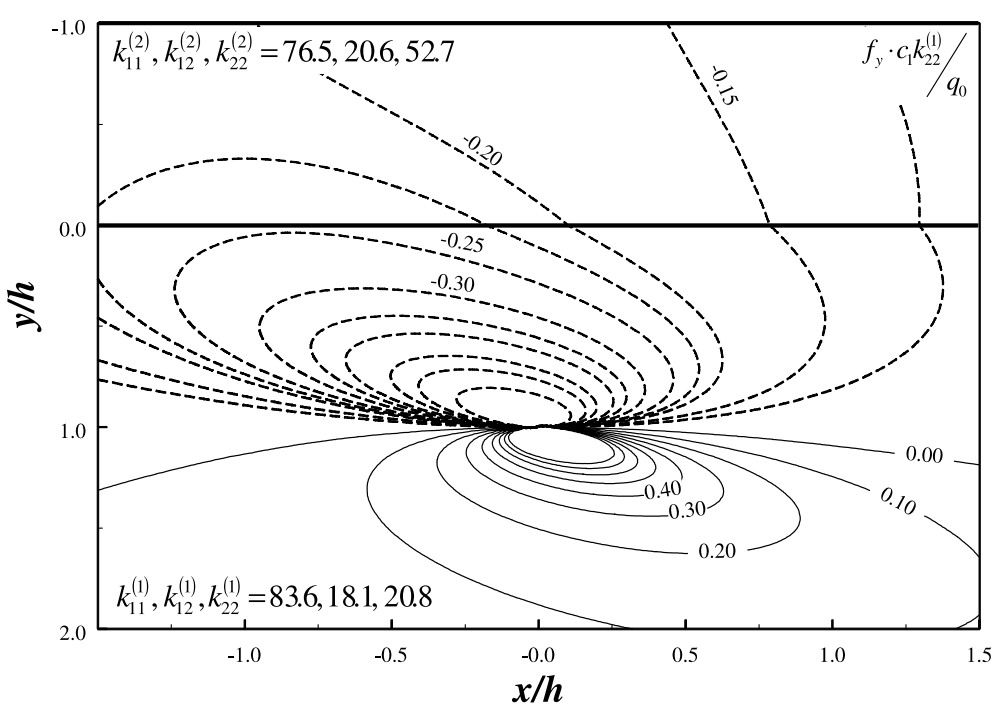

Fig. 7. Heat flux in the $y$-direction of the thin-layer medium with a concentrated heat source applied in the half-plane.

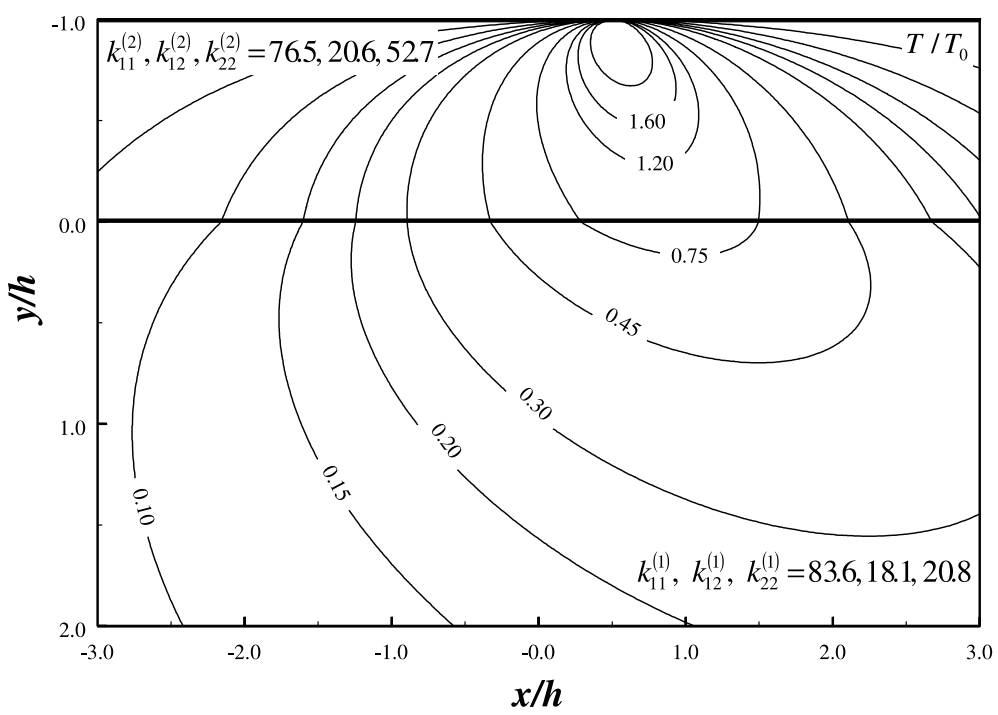

Fig. 8. Temperature distribution of the thin-layer medium with a concentrated temperature applied on the top surface.

\section{Conclusion}

A linear coordinate transformation method is introduced to solve the heat conduction problem of a thin-layer anisotropic medium subjected to arbitrary thermal loadings applied in the medium or on the boundary surface. With this linear coordinate transformation, the original anisotropic thin-layer problem is reduced to an equivalent isotropic problem without complicating the boundary and interface continuity conditions. By using the Fourier transform technique and a series expansion, analytical solutions for temperature and heat flux are presented in an explicit form. A computational program for numerical calculation of the full-field distribution is easily constructed by using the explicit formulation of the solutions. It is evident from numerical results that the material anisotropy has a marked influence on the distribution of temperature and heat flux fields. The linear coordinate transformation method developed in this 


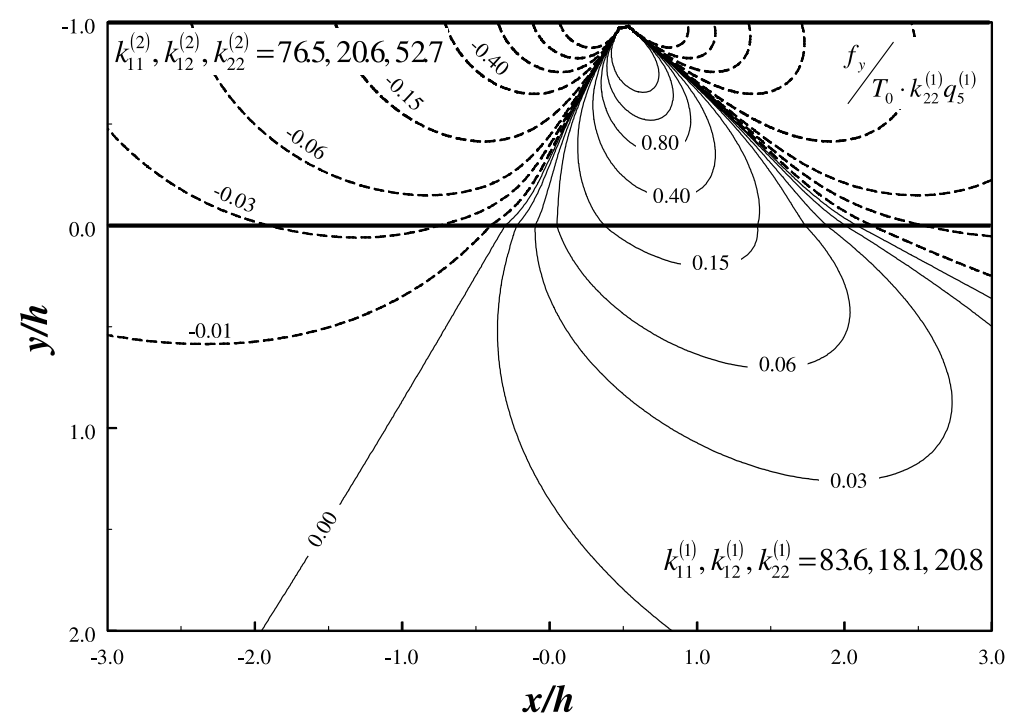

Fig. 9. Heat flux in the $y$-direction of the thin-layer medium with a concentrated temperature applied on the top surface.

article effectively solves the heat conduction problem in thin-layer anisotropic media. In fact, this method can be extended to solve more complicated problems and will be given in a follow-up article.

\section{Acknowledgements}

The financial support of the authors from the National Science Council, Republic of China, though Grant NSC 872218-E002-022 to National Taiwan University is gratefully acknowledged.

\section{References}

[1] M.N. Ozisik, Heat Conduction, Wiley, New York, 1980.

[2] Y.I. Mindolin, A.I. Uzdalev, Anisotropic plate under influence of distributes and point heat sources, Mech. Solids 30 (1995) 118123.

[3] B. Sharma, Thermal stresses in transversely isotropic semi-infinite elastic solids, J. Appl. Mech. 25 (1958) 86-88.

[4] T.R. Tauchert, A.Y. Akoz, Thermal stresses in an orthotropic elastic slab due to prescribed surface temperatures, J. Appl. Mech. 41 (1974) 222-228.

[5] T.R. Tauchert, A.Y. Akoz, Stationary temperature and stress fields in an anisotropic elastic slab, J. Appl. Mech. 42 (1975) 647650.

[6] G.P. Mulholland, B.P. Gupta, Heat transfer in a three-dimensional anisotropic solid of arbitrary shape, J. Heat Transfer 99 (1977) $135-137$.

[7] Y.P. Chang, Analytical solution for heat conduction in anisotropic media in infinite semi-infinite, and two-place-bounded regions, Int.J. Heat Mass Transfer 20 (1977) 1019-1028.

[8] K.C. Poon, Transformation of heat conduction problems in layered composites from anisotropic to orthotropic, Lett. Heat Mass Transfer 6 (1979) 503-511.

[9] K.C. Poon, R.C.H. Tsou, Y.P. Chang, Solution of anisotropic problems of first class by coordinate-transformation, J. Heat Transfer 101 (1979) 340-345.

[10] X.Z. Zhang, Steady-state temperatures in an anisotropic strip, J. Heat Transfer 112 (1990) 16-20.

[11] L. Yan, A.H. Sheikh, J.V. Beck, Thermal characteristics of two-layered bodies with embedded thin-film heat source, J. Electron. Packag. 115 (1993) 276-283.

[12] S. Thangjitham, H.J. Choi, Thermal stresses in a multilayered anisotropic medium, J. Appl. Mech. 58 (1991) $1021-1027$. 